\title{
Elevated D-dimer increases the risk of dialysis after surgery in patients with Stanford A aortic dissection through the impact of the coagulation system
}

\author{
Lu Han ${ }^{1,2,3 \#}$, Lu Dai ${ }^{2,3,4 \#}$, Hai-Yang Li ${ }^{2,3,4}$, Feng Lan ${ }^{2,3,4,5}$, Wen-Jian Jiang ${ }^{2,3,4,5}$, Hong-Jia Zhang ${ }^{2,3,4,5}$ \\ ${ }^{1}$ Department of Cardiac Surgery, Beijing Shijitan Hospital, Capital Medical University, Beijing 100038, China; ${ }^{2}$ Department of Cardiac Surgery, \\ Beijing Anzhen Hospital, Capital Medical University, Beijing 100029, China; ${ }^{3}$ Beijing Lab for Cardiovascular Precision Medicine, Beijing 100069, \\ China; ${ }^{4}$ Beijing Institute of Heart, Lung and Blood Vessel Diseases, Beijing 100029, China; ${ }^{5}$ Beijing Aortic Disease Center, Cardiovascular Surgery \\ Center, Beijing 100029, China \\ \#These authors contributed equally to this work. \\ Correspondence to: Wen-Jian Jiang, MD; Hong-Jia Zhang, PhD, MD. Department of Cardiac Surgery, Beijing Anzhen Hospital, Capital Medical \\ University, Beijing 100029, China. Email: jiangwenjian@ccmu.edu.cn; zhanghongjia722@hotmail.com.
}

Background: To investigate whether Elevated D-dimer increases the risk of dialysis after surgery in patients with Stanford A aortic dissection.

Methods: A total of 120 patients with type A aortic dissection who underwent surgery at our institution from August 2014 to December 2015 were enrolled in the study. Acute type A aortic dissection was treated with Sun's operation. Blood samples were collected before anesthesia induction, 4 hours after surgery, and 24 hours after surgery. Patients were divided into two groups according to their D-dimer levels. Group A had D-dimer concentrations below 3,000 $\mu \mathrm{g} / \mathrm{L}$; Group B had D-dimer concentrations above 3,000 $\mu \mathrm{g} / \mathrm{L}$.

Results: Group A ( $=99 ; 82.5 \%$ of total patients) had D-dimer levels below 3,000 $\mu \mathrm{g} / \mathrm{L}$. Eighteen patients in Group A (18.18\%) died within 30 days after surgery. Group B (n=21; $18.5 \%$ of total patients) had D-dimer levels above 3,000 $\mu \mathrm{g} / \mathrm{L}$. Seven patients in Group B (28\%) died within 30 days after surgery. A significantly higher percentage of patients in Group B had acute renal failure and the application of continuous renal replacement therapy $(\mathrm{P}=0.02)$. There were significant differences between the groups in intraoperative blood loss $(\mathrm{P}=0.001)$ and hemostatic drugs administered, such as intraoperative prothrombin complex $(\mathrm{P}=0.015)$. The D-dimer $(\mathrm{P}<0.001)$, FIB $(\mathrm{P}=0.008)$ and FDP $(\mathrm{P}<0.001)$ in the $\mathrm{B}$ group were significantly higher than those in the A group, but there was no significant difference between the 4 hours after the operation and the 24 hours after the operation. Thromboelastogram (TEG) examination showed that preoperative $\mathrm{R}$ in group B was shorter than the A group, 4 hours after operation was still lower in group B than in group A. Through ROC analysis, D-dimer is a prognostic indicator for postoperative renal failure. When cut-off $=1,039.00$, sensitivity $=91.7 \%$, specificity $=54.2 \%$. When D-dimer is below $1,039 \mu \mathrm{g} / \mathrm{L}$, the risk of dialysis after surgery in patients with Stanford A aortic dissection is low. Prognostic value of D-dimer was evaluated using ROC analysis and the results showed that the area under curve (AUC) of D-dimer as prognostic indicator for postoperative renal failure was 0.741 (95\% CI, 0.642-0.840; $\mathrm{P}$ value $<0.001$ ). Stepwise binary logistic regression analysis revealed that total suspended red, wakefulness delayed, D-dimer were independent predictors of dialysis risk after surgery in patients with Stanford A aortic dissection among all the other factors.

Conclusions: D-dimer above 3,000 $\mu \mathrm{g} / \mathrm{L}$ increases the risk of dialysis after surgery in patients with Stanford A aortic dissection through the impact of the coagulation system. When D-dimer is below 1,039 $\mu \mathrm{g} / \mathrm{L}$, the risk of dialysis after surgery in patients with Stanford A aortic dissection is low.

Keywords: D-dimmer; aortic dissecting aneurysm; Stanford A; dialysis

Submitted Jul 17, 2018. Accepted for publication Nov 19, 2018.

doi: $10.21037 /$ jtd.2018.11.138

View this article at: http://dx.doi.org/10.21037/jtd.2018.11.138

(C) Journal of Thoracic Disease. All rights reserved.

jtd.amegroups.com

J Thorac Dis 2018;10(12):6783-6793 


\section{Introduction}

Up-to-date data on the epidemiology of Stanford type A aortic dissection are scarce. The Oxford Vascular Study estimated the incidence of aortic dissection at six per 100,000 persons per year. Aortic dissection requires emergency diagnosis and treatment (1). The reported mortality rate with surgical treatment is as high as $10 \%$ and the complication rate is $30 \%$ (2). Coagulation function in the perioperative period is a key factor affecting surgical outcomes (3). There are many methods to evaluate the coagulation mechanism, all of which have limitations.

Renal dysfunction is a common complication of aortic dissection after surgery, in addition to part of patients spontaneously, most patients with renal insufficiency requiring dialysis treatment, postoperative dialysis process may lead to problems of circulation instability, infection, thrombosis, and ion disturbance can affect postoperative recovery of patients, and even cause the death of the patient. There has been a lot of research into the protection of renal function after aortic dissection, but whether the increase in D-dimer before surgery increases the risk of postoperative dialysis remains unknown.

$\mathrm{D}$-dimer is a specific degradation product produced by the hydrolysis of fibrin monomers after they are crosslinked with factor XIII. D-dimer is derived from plasminsolubilized cross-linked fibrin clots. D-dimer concentrations are primarily used to exclude pulmonary embolism and in the auxiliary diagnosis of acute aortic dissection (4). D-dimer elevation is very common in aortic dissection patients, with some patients having levels higher than $3,000 \mu \mathrm{g} / \mathrm{L}$. Aortic dissection requires emergency surgery; however, the effect of elevated D-dimer on intraoperative and postoperative outcomes has not been reported. In this study, we evaluated whether elevated D-dimer increases dialysis risk after surgery in patients with Stanford A aortic dissection and its mechanisms (5).

\section{Methods}

\section{Study design}

A total of 120 patients with type A aortic dissection who underwent surgery at our institution from August 2014 through December 2015 were enrolled in the study. Acute type A aortic dissection was treated with Sun's operation (ascending aorta replacement or Bentall + aortic arch replacement + descending aorta stenting). The average patient age was $48.08 \pm 10.73$ years. Patients were divided into two groups according to their D-dimer levels. Group A had D-dimer concentrations below 3,000 $\mu \mathrm{g} / \mathrm{L}$; group B had D-dimer concentrations above 3,000 $\mu \mathrm{g} / \mathrm{L}$. The characteristics of each group at anesthetic induction are shown in Table 1.

\section{Surgical procedures}

Patients with acute type A aortic dissection underwent emergency operation within $24 \mathrm{~h}$ of admission. Under general anesthesia, free right axillary artery and femoral artery reserve, thoracic incision, thoracic layer, free innominate vein and brachiocephalic vessels. The pericardium, extracorporeal circulation right axillary artery or right axillary artery and femoral artery and right atrial cannulation, left ventricular drainage, blocking the aorta, the aortic incision, according to the ascending aorta aortic valve for replacement or Bentall, nasopharyngeal temperature and rectal temperature to 23 to $25^{\circ} \mathrm{C}$, blocking the brachiocephalic vessels after opening aorta, selection $5 \mathrm{~mL} /(\mathrm{kg} \cdot \mathrm{min})$ perfusion, descending thoracic aortic stent implantation (Memory sent), and after surgery or retain the classic SUN arch surgery. Application of right ventricular bypass with aortic wall and pericardium after open circulation. Cardiopulmonary bypass time, deep hypothermic circulatory arrest time, and other intraoperative Characteristics are shown in Table 2.

\section{Blood collection}

To examine the effect of aortic dissection and surgery on D-dimer concentration, blood samples were obtained from all patients at three different time points: at induction of anesthesia, 4 hours postoperatively, and 24 hours postoperatively. The first $5 \mathrm{~mL}$ of blood drawn were discarded to eliminate the dilution effect of saline solution. Blood samples were stored in citrated blood collection tubes. Blood was obtained from the central venous catheter or a peripheral vein and anticoagulated with sodium citrate for measurement of coagulation and fibrinolysis. Blood samples were centrifuged for $15 \mathrm{~min}$ at $3,500 \mathrm{rpm}$ at $4{ }^{\circ} \mathrm{C}$ and frozen at $-80{ }^{\circ} \mathrm{C}$ until assayed.

\section{Hemostatic system assays}

International normalized ratio, fibrin degradation products, hemoglobin level, platelet counts, and fibrinogen concentration were assayed on an automated blood coagulation analyzer. Specific assays were performed to 
Table 1 Patient characteristic according to group at time of surgery

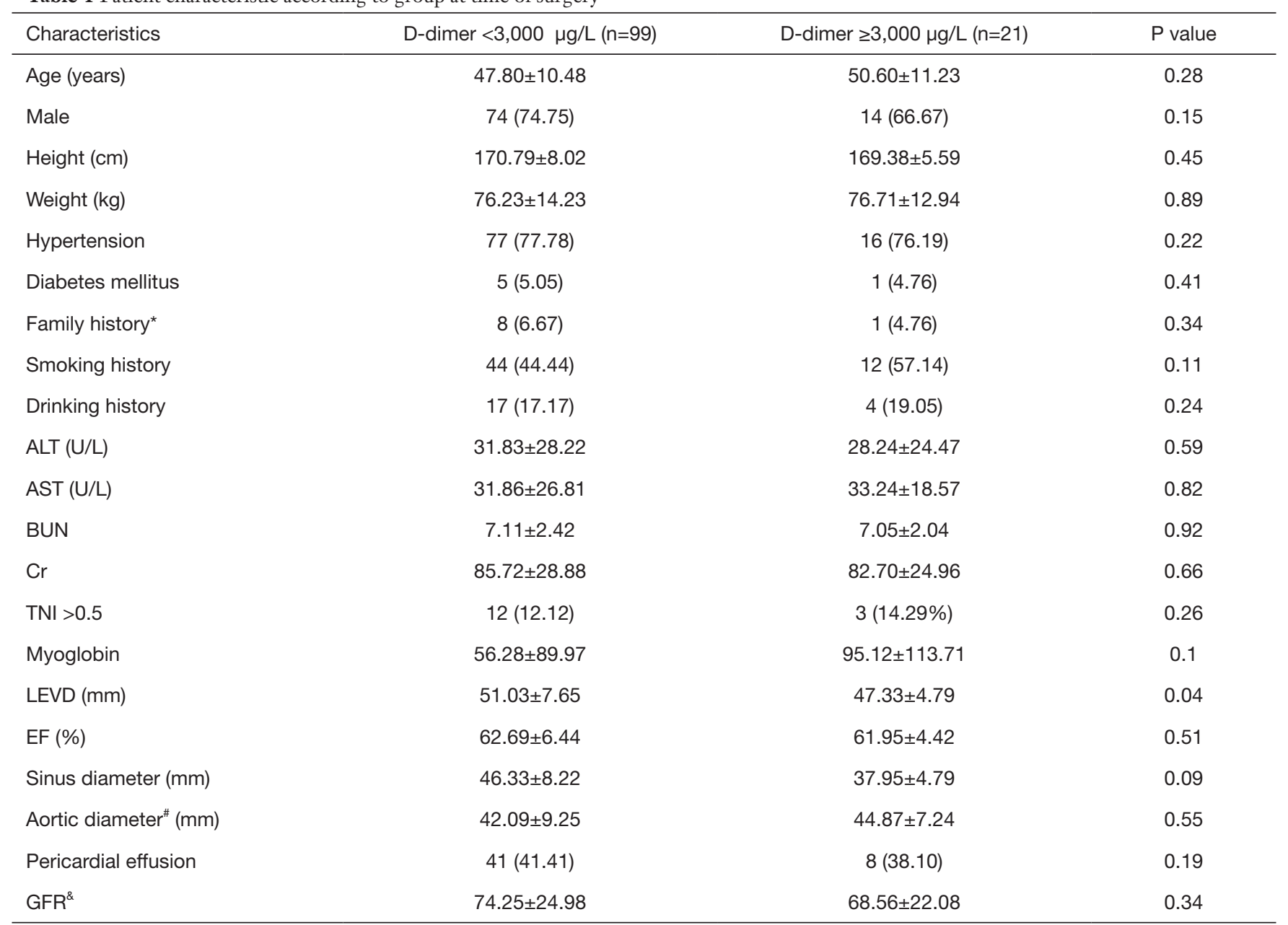

Values are mean $\pm \mathrm{SD}$ or $\mathrm{n}(\%)$. *, relatives with a history of dissection or unexplained sudden death. *, ascending aorta diameter. ${ }^{\text {, }}$, Glomerular filtration rate $\left(\mathrm{mL} / \mathrm{min} / 1.73 \mathrm{~m}^{2}\right)$. LEVD, left ventricular end diastolic diameter.

assess the activation of coagulation, anticoagulation, and fibrinolysis. Plasma was assayed with the monoclonal antibody sandwich ELISA technique. All ELISA assays were performed twice and the mean value was used for analysis.

\section{The state of the preoperative renal artery}

Through the observation of chest and abdomen CTA, it was found that the state of the preoperative renal artery whether the renal artery was a true lumen or a false lumen blood supply. We indicated the number of patients with their renal arteries involved in the dissection, and the number of patients with obstruction or stenosis of the renal arteries.

\section{Statistical methods}

The data are presented as the mean and standard deviation. SPSS version 19 was used for statistical analysis. GraphPad Prism 5.0 (La Jolla, CA) was used for the graph production. The $t$ test was used to compare data between the two groups, and the data were analyzed with ANOVA. $\mathrm{P}<0.05$ was considered statistically significant. The Prognostic value of D-dimer was evaluated using respective Receiver Operating Characteristic (ROC) curves. Stepwise binary logistic regression analysis was used to analyze the independent factors affecting the risk of dialysis after surgery in patients with Stanford A aortic dissection. 
Table 2 Intraoperative characteristics

\begin{tabular}{|c|c|c|c|}
\hline Characteristics & D-dimer <3,000 $\mu \mathrm{g} / \mathrm{L}(\mathrm{n}=99)$ & D-dimer $\geq 3,000 \mu \mathrm{g} / \mathrm{L}(\mathrm{n}=21)$ & $P$ value \\
\hline Aortic occlusion time (min) & $123.55 \pm 45.27$ & $121.24 \pm 29.15$ & 0.82 \\
\hline Stop cycle time (min) & $26.96 \pm 9.99$ & $27.19 \pm 6.49$ & 0.92 \\
\hline The lowest nasal temperature $\left({ }^{\circ} \mathrm{C}\right)$ & $22.98 \pm 2.03$ & $22.64 \pm 1.18$ & 0.46 \\
\hline Operation time (h) & $8.26 \pm 1.95$ & $9.0 \pm 1.50$ & 0.1 \\
\hline Intraoperative blood loss* (mL) & $1,387.88 \pm 556.46$ & $1,980.95 \pm 948.48$ & 0.001 \\
\hline Postoperative total drainage ${ }^{\star \star}$ & $216.88 \pm 1,247.10$ & $2,985.23 \pm 2,091.62$ & 0.02 \\
\hline Red blood cell transfusion (mL) & $1,359.09 \pm 1,384.23$ & $1,592.86 \pm 1,122.97$ & 0.02 \\
\hline Protamine (mg) & $476.53 \pm 123.82$ & $459.52 \pm 137.49$ & 0.58 \\
\hline Intraoperative fibrinogen (g) & $1.68 \pm 1.12$ & $2.19 \pm 1.29$ & 0.07 \\
\hline Intraoperative prothrombin complex & $652.53 \pm 427.22$ & $914.29 \pm 519.89$ & 0.015 \\
\hline VIIFactor & $10(10.1)$ & $4(19.0)$ & 0.14 \\
\hline
\end{tabular}

Values are mean \pm SD or $\mathrm{n}(\%) .{ }^{*}$, estimated according to use of intraoperative gauze. ${ }^{\star *}$, postoperative drainage less than $100 \mathrm{~mL}$ in $24 \mathrm{~h}$ after extubation. Postoperative drainage was calculated. Surgical bleeding was excluded.

\section{Results}

\section{Preoperative situation}

In the course of the diagnosis and treatment of patients with Stanford A aortic dissection, some patients had elevated D-dimer significantly, with varying degrees of elevation and some even exceeding 20,000 $\mu \mathrm{g} / \mathrm{L}$. Through the preoperative situation analysis can be found group A $(\mathrm{n}=99 ; 82.5 \%$ of total patients) had D-dimer levels below $3,000 \mu \mathrm{g} / \mathrm{L}$. Among them, $74(74.75 \%)$ males, with an average age of $47.80 \pm 10.48$, weight $76.23 \pm 14.23 \mathrm{~kg}$, EF $(62.69 \pm 6.44) \%$, and TNI $>0.5$ were $12(12.12 \%)$; group B $(\mathrm{n}=21 ; 18.5 \%$ of total patients) had D-dimer concentrations above 3,000 $\mu \mathrm{g} / \mathrm{L}$. Fourteen (66.67\%) males, with an average age of $50.60 \pm 11.23$, weight $76.71 \pm 12.94 \mathrm{~kg}$, EF $(61.95 \pm 4.42) \%$, and TNI $>0.5$ were $3(14.29 \%)$. The LEVD at the preoperative situation in group A was significantly higher than that in group $B(P=0.04)$. The basic information of the other two groups before operation was basically the same.

\section{Intraoperative condition}

Cardiopulmonary bypass time, aortic occlusion time, and stop cycle time (selective cerebral perfusion time) in group A were $208.67 \pm 58.80,123.55 \pm 45.27$, and $26.96 \pm 9.99 \mathrm{~min}$, in group B were $215.42 \pm 44.58,121.24 \pm 29.15$, and $27.19 \pm 6.49 \mathrm{~min}$, respectively. There were no significant differences between the groups in the time of cardiopulmonary bypass $(\mathrm{P}=0.62)$, the time of aortic occlusion $(\mathrm{P}=0.82)$, or the time of stop cycle time $(\mathrm{P}=0.92)$.

However, there were significant differences between the groups in intraoperative blood loss $(\mathrm{P}=0.001)$, total postoperative drainage volume $(\mathrm{P}=0.02)$, and hemostatic drugs administered such as intraoperative prothrombin complex $(\mathrm{P}=0.015)$, with group $\mathrm{B}$ having significantly higher than group $\mathrm{A}$. Intraoperative $\mathrm{RBC}(\mathrm{P}=0.02)$, plasma $(\mathrm{P}<0.001)$ and platelet transfusion $(\mathrm{P}<0.001)$ in group $\mathrm{B}$ were significantly higher than those in group $\mathrm{A}$. There were no significant differences between the groups in protamine $(\mathrm{P}=0.58)$ or fibrinogen $(\mathrm{P}=0.07)$, prothrombin complex $(\mathrm{P}=0.015)$, VIIFactor $(\mathrm{P}=0.14)$ administration, because these are routinely given to regulate clotting.

\section{Analysis of blood samples and thromboelastogram (TEG)}

Blood samples were collected at induction of anesthesia, 
Table 3 Changes in coagulation indexes during and after surgery

\begin{tabular}{|c|c|c|c|}
\hline Characteristics & D-dimer $<3,000 \mu \mathrm{g} / \mathrm{L}(\mathrm{n}=99)$ & $D$-dimer $\geq 3,000 \mu g / L(n=21)$ & $P$ value \\
\hline Anesthesia induction & $1,196.18 \pm 853.06$ & $17,650.1 \pm 22,362.5$ & $<0.001$ \\
\hline 4 hours after operation & $4,252.69 \pm 5,000.15$ & $3,307.9 \pm 2,804.9$ & 0.46 \\
\hline 24 hours after operation & $3,388.08 \pm 3,667.94$ & $3,471.1 \pm 2,901.1$ & 0.92 \\
\hline Anesthesia induction & $1.05 \pm 1.59$ & $1.07 \pm 0.09$ & 0.39 \\
\hline 4 hours after operation & $1.39 \pm 1.79$ & $1.12 \pm 0.14$ & 0.51 \\
\hline 24 hours after operation & $1.17 \pm 0.15$ & $1.15 \pm 0.19$ & 0.52 \\
\hline \multicolumn{4}{|l|}{ FIB (g/L) } \\
\hline 24 hours after operation & $3.51 \pm 1.25$ & $3.14 \pm 0.80$ & 0.19 \\
\hline \multicolumn{4}{|l|}{ FDP (mg/L) } \\
\hline Anesthesia induction & $15.57 \pm 13.38$ & $128.97 \pm 126.66$ & $<0.001$ \\
\hline 4 hours after operation & $42.23 \pm 45.00$ & $32.35 \pm 24.03$ & 0.33 \\
\hline 24 hours after operation & $35.34 \pm 30.31$ & $32.97 \pm 24.42$ & 0.74 \\
\hline \multicolumn{4}{|l|}{ HGB (g/L) } \\
\hline Anesthesia induction & $135.32 \pm 17.24$ & $135.38 \pm 18.10$ & 0.99 \\
\hline 4 hours after operation & $97.53 \pm 20.09$ & $90.35 \pm 18.80$ & 0.14 \\
\hline
\end{tabular}

Values are mean \pm SD. INR, international normalized ratio; FIB, fibrinogen; FDP, fibrin degradation products; HGB, hemoglobin; PLT, platelet.

4 hours postoperatively, and 24 hours postoperatively. The D-dimer $(\mathrm{P}<0.001)$, FIB $(\mathrm{P}=0.008)$ and FDP $(\mathrm{P}<0.001)$ in the $\mathrm{B}$ group were significantly higher than those in the A group, but there was no significant difference between the 4 hours after the operation and the 24 hours after the operation (Table 3).

TEG examination showed that preoperative $\mathrm{R}$ in group B was shorter than group A, 4 hours after operation was still lower in group B than in group A. But there was no significant difference between the two groups at 24 hours after operation (Table 4).

\section{Postoperative condition}

Eighteen patients in Group A (18.2\%) died within 30 days after surgery and seven patients in group B (33.3\%) died within 30 days after surgery $(\mathrm{P}=0.120)$. A significantly higher percentage of patients in group B $(8,38.1 \%)$ than in group $\mathrm{A}(16,16.2 \%)$ had acute renal failure and the application of continuous renal replacement therapy $(\mathrm{P}=0.022)$. Postoperative cerebral infarction $(\mathrm{P}=0.069)$, respiratory failure $(\mathrm{P}=0.402)$, paraplegia $(\mathrm{P}=0.956)$, ICU time $(\mathrm{P}=0.386)$ and hospitalization time $(\mathrm{P}=0.980)$ were not obviously abnormal in the two groups (Table 5). 
Table 4 Thromboelastogram

\begin{tabular}{|c|c|c|c|}
\hline Characteristics & D-dimer <3,000 $\mu \mathrm{g} / \mathrm{L}(\mathrm{n}=99)$ & D-dimer $\geq 3,000 \mu \mathrm{g} / \mathrm{L}(\mathrm{n}=21)$ & $P$ value \\
\hline \multicolumn{4}{|l|}{$\mathrm{R}(\min )$} \\
\hline Anesthesia induction & $7.02 \pm 1.20$ & $5.12 \pm 1.34$ & 0.02 \\
\hline 4 hours after operation & $8.32 \pm 2.40$ & $6.58 \pm 2.21$ & 0.03 \\
\hline 24 hours after operation & $5.97 \pm 2.20$ & $6.09 \pm 1.40$ & 0.82 \\
\hline Anesthesia induction & $1.71 \pm 1.11$ & $1.87 \pm 0.92$ & 0.55 \\
\hline 4 hours after operation & $2.87 \pm 1.96$ & $3.12 \pm 2.12$ & 0.6 \\
\hline 24 hours after operation & $2.27 \pm 1.49$ & $2.41 \pm 0.98$ & 0.7 \\
\hline \multicolumn{4}{|l|}{ Angle $(\alpha)$} \\
\hline 24 hours after operation & $62.19 \pm 11.75$ & $61.22 \pm 7.99$ & 0.74 \\
\hline \multicolumn{4}{|l|}{ Maximum clot strength } \\
\hline Anesthesia induction & $62.87 \pm 7.44$ & $59.81 \pm 10.06$ & 0.12 \\
\hline 4 hours after operation & $54.76 \pm 10.58$ & $52.93 \pm 11.15$ & 0.5 \\
\hline 24 hours after operation & $56.77 \pm 11.03$ & $55.51 \pm 8.87$ & 0.64 \\
\hline
\end{tabular}

$\mathrm{R}$, Clotting factor response time; K, Angle ( $\alpha$ ), Aggregation velocity parameter of blood clot.

Table 5 Postoperative characteristics

\begin{tabular}{|c|c|c|c|}
\hline Characteristics & D-dimer $<3,000 \mu \mathrm{g} / \mathrm{L}(\mathrm{n}=99)$ & D-dimer $\geq 3,000 \mu \mathrm{g} / \mathrm{L}(\mathrm{n}=21)$ & $P$ value \\
\hline Respiratory failure & $16(16.2)$ & $5(23.8)$ & 0.402 \\
\hline Ventilator time & $77.39 \pm 130.42$ & $145.71 \pm 262.11$ & 0.257 \\
\hline Dialysis* & $16(16.2)$ & $8(38.1)$ & $0.022^{*}$ \\
\hline ICU time (d) & $5.00 \pm 6.20$ & $6.32 \pm 6.84$ & 0.386 \\
\hline Hospitalization time & $15.91 \pm 8.48$ & $15.86 \pm 7.95$ & 0.980 \\
\hline Mortality within 30 days after surgery & $18(18.2)$ & $7(33.3)$ & 0.120 \\
\hline
\end{tabular}

Values are mean \pm SD or $n(\%) .{ }^{*}$, application of dialysis in the treatment of renal failure.

\section{The state of the preoperative renal artery}

Preoperative thoracic and abdominal CTA showed that 99 patients with $\mathrm{D}$-dimer $<3,000 \mu \mathrm{g} / \mathrm{L}$ had left renal artery involvement in 7 cases, right renal artery involvement in 5 cases and bilateral renal artery involvement in 3 cases. There were 21 patients with D-dimer $>3,000 \mu \mathrm{g} / \mathrm{L}, 2$ with left renal artery involvement, 1 with right renal artery involvement and 1 with bilateral renal artery involvement. Among 54 patients with D-dimer $<1,039 \mu \mathrm{g} / \mathrm{L}, 5$ had left renal artery involvement, 3 had right renal artery involvement and 2 had bilateral renal artery involvement. There were 66 patients with $\mathrm{D}$-dimer $>1,039 \mu \mathrm{g} / \mathrm{L}$, 4 patients with left renal artery involvement, 3 patients with right renal artery involvement and 2 patients with 


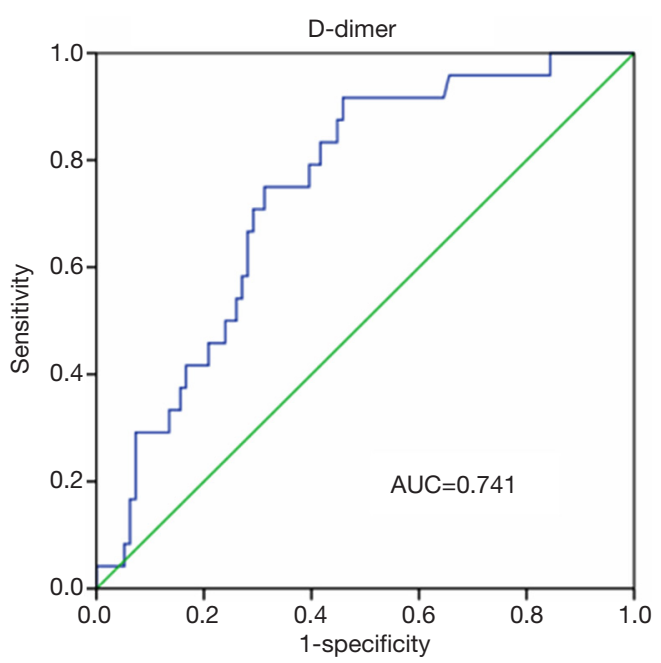

Figure $1 \mathrm{D}$-dimer as a prognostic indicator for postoperative renal failure $(\mathrm{AUC}=0.741, \mathrm{P}<0.001)$. When cut-off $=1,039.00$, sensitivity $=91.7 \%$, specificity $=54.2 \%$.

bilateral renal artery involvement. The above data were statistically analyzed and found no statistical significance. Through thoracoabdominal CTA, it is difficult to observe the number of patients with obstruction or stenosis of the renal arteries.

\section{ROC analysis}

Prognostic value of D-dimer was evaluated using ROC analysis. The results showed that the area under curve (AUC) of D-dimer as prognostic indicator for postoperative renal failure was 0.741 (95\% CI, 0.642-0.840; $\mathrm{P}$ value $<0.001)$. When a $\mathrm{D}$-dimer value of 1,039 was used as the cutoff, the corresponding sensitivity and specificity for distinguishing the high risk of dialysis after surgery in patients with Stanford A aortic dissection from low risk were $91.7 \%$ and $54.2 \%$, respectively (Figure 1). D-dimer as prognostic indicator for other prognostic indicators was showed in Figure 2. Postoperative respiratory failure ( $\mathrm{AUC}=0.644)$; postoperative gastrointestinal bleeding (AUC=0.709); Multiple organ failure (AUC=0.737); and poor prognosis $(\mathrm{AUC}=0.629)$ (Poor prognosis = death + digestive tract hemorrhage + cerebral infarction + cerebral hemorrhage + deep coma + conscious delay + myocardial infarction + heart failure + respiratory failure + renal failure + multiple organ failure).

\section{Comparison of different groups of patients with renal insufficiency requiring dialysis}

We used a D-dimer value of 3,000 $\mu \mathrm{g} / \mathrm{L}$ to group patients by experience. Through ROC curve analysis, the most instructive cut-off value of $\mathrm{D}$-dimer for renal insufficiency patients requiring dialysis is $1,039 \mu \mathrm{g} / \mathrm{L}$. When the value of D-dimer is lower than $1,039 \mu \mathrm{g} / \mathrm{L}$, the lower probability of renal insufficiency patients requiring dialysis is found (Figure 3).

\section{Multivariable logistic analysis}

Among the factors that may affect renal failure after aortic dissection (total red blood transfusion, total plasma transfusion, total platelet transfusion, intraoperative dosage of fibrinogen, intraoperative dosage of prothrombin complex; delayed awakening, infection, respiratory failure, heart failure, intraoperative blood loss, operation time hours, dimer group), total suspended red (OR=1.001; 95\% CI, 1.000-1.001), wakefulness delayed (OR=16.601; 95\% CI, 3.224-85.477) and D-dimer (OR=10.292; 95\% CI, 1.785-59.340; D-dimer=1,039) were independent predictors of dialysis risk after surgery in patients with Stanford A aortic dissection. According to the comparison of the two groups after operation when D-dimer was higher or lower than $1,039 \mu \mathrm{g} / \mathrm{L}$ (Tables 6,7).

\section{Discussion}

Renal dysfunction is one of the common complications after aortic dissection, the reason for large doses of vasopressors, renal hypoperfusion, massive transfusion, dissection false lumen involvement and other reasons. Large dose of vasopressors, renal hypoperfusion, massive transfusion are related to poor coagulation mechanisms. D-dimer is a commonly used clinical index to exclude pulmonary embolism and has been used as a diagnostic tool in aortic dissection patients. However, studies evaluating D-dimer in aortic dissection patients who undergo surgery have not been published (6). This study found that different levels of D-dimer before surgery can lead to changes in coagulation mechanisms during surgery, resulting in an increase in blood transfusion and use of hemostatic agents, resulting in acute renal dysfunction and requiring dialysis treatment.

In Stanford type A aortic dissection surgery, intraoperative 
A

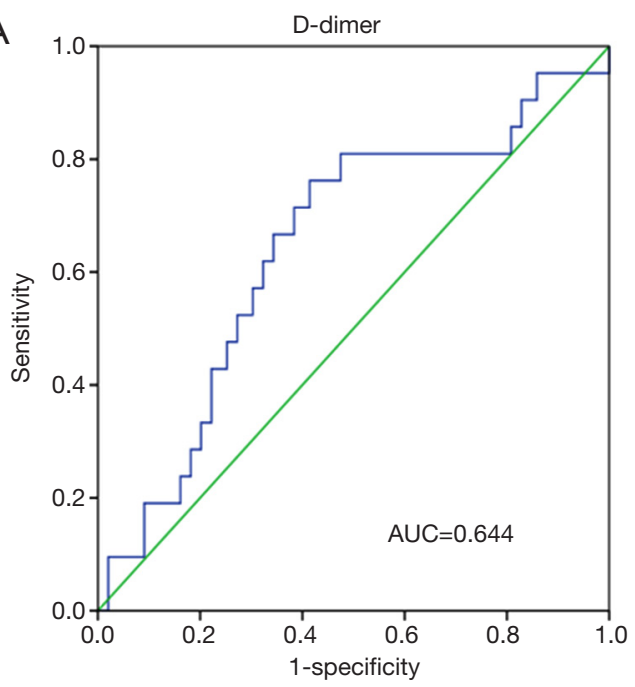

C

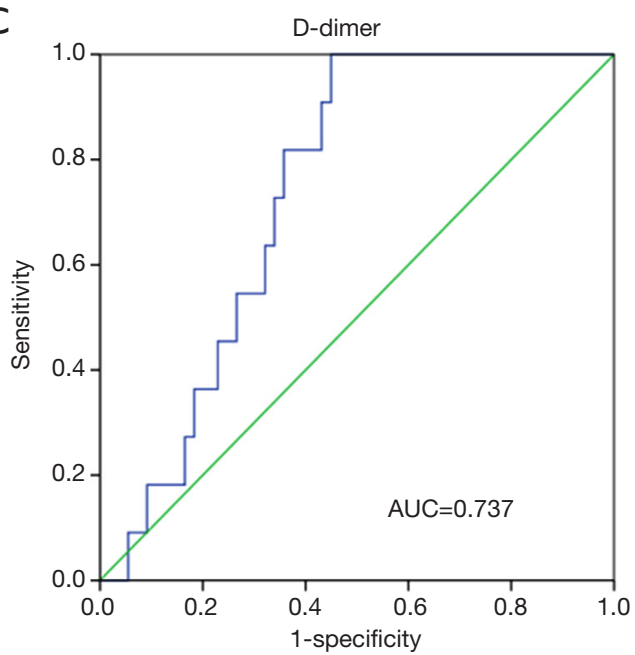

B

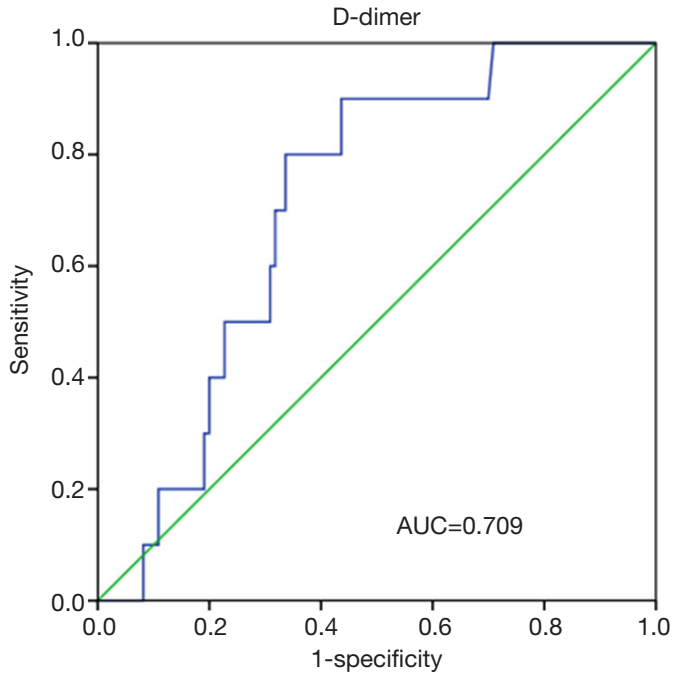

D

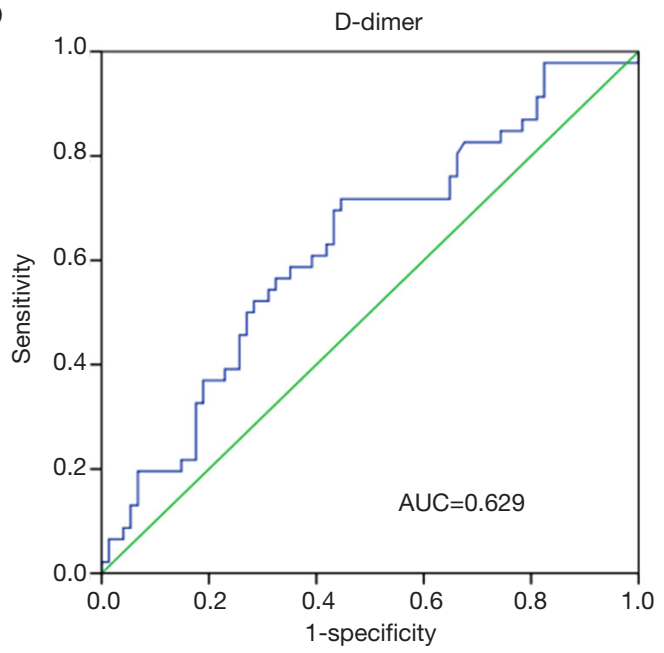

Figure $2 \mathrm{D}$-dimer as a prognostic indicator for postoperative respiratory failure (A), $\mathrm{AUC}=0.644$; postoperative gastrointestinal bleeding (B), $\mathrm{AUC}=0.709$; multiple organ failure (C) $\mathrm{AUC}=0.737$; and poor prognosis (D) $\mathrm{AUC}=0.629$. Poor prognosis = death + digestive tract hemorrhage + cerebral infarction + cerebral hemorrhage + deep coma + conscious delay + myocardial infarction + heart failure + respiratory failure + renal failure + multiple organ failure.
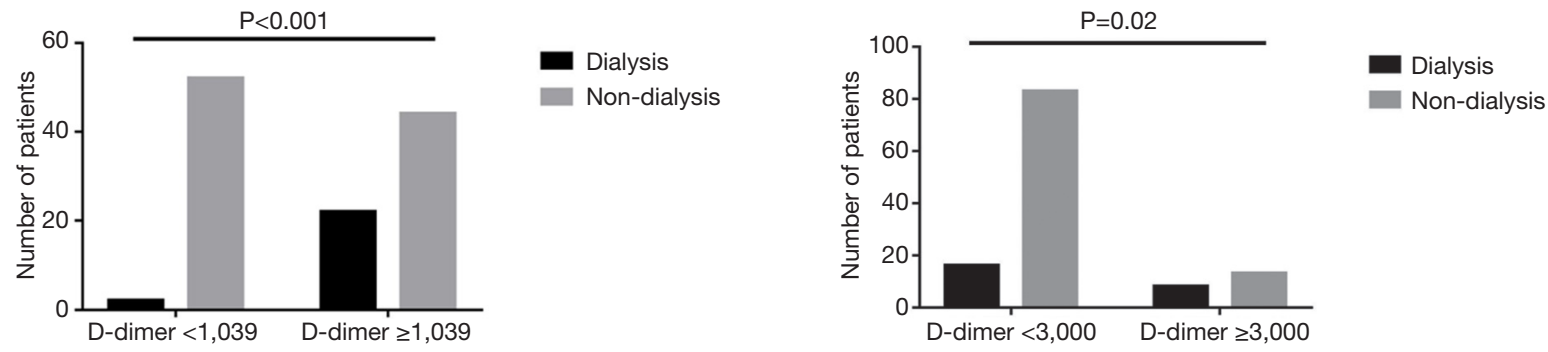

Figure 3 According to the different data of $\mathrm{D}$-dimer, when $\mathrm{D}$-dimer is 1,039 and $3,000 \mu \mathrm{g} / \mathrm{L}$, it has statistical significance $(\mathrm{P}<0.001, \mathrm{P}=0.02)$. Through the statistical results, it can be seen that when D-dime is less than $1,039 \mu \mathrm{g} / \mathrm{L}$, the incidence of dialysis is lower. 
Table 6 Multiple factors analysis of different factors on dialysis induced by renal

\begin{tabular}{|c|c|c|c|c|c|c|c|}
\hline Characteristics & $B$ & S.E. & Wald & df & Sig. & $\operatorname{Exp}(\mathrm{B})$ & $95 \% \mathrm{Cl}$ for EXP (B) \\
\hline \multicolumn{8}{|l|}{ Step 1} \\
\hline Wakefulness delayed & 3.267 & 0.715 & 20.849 & 1 & 0 & 26.231 & $6.453-106.619$ \\
\hline Constant & -1.968 & 0.296 & 44.159 & 1 & 0 & 0.14 & \\
\hline \multicolumn{8}{|l|}{ Step 2} \\
\hline Wakefulness delayed & 2.98 & 0.775 & 14.781 & 1 & 0 & 19.682 & $4.309-89.903$ \\
\hline Constant & -3.434 & 0.564 & 37.056 & 1 & 0 & 0.032 & \\
\hline \multicolumn{8}{|l|}{ Step 3} \\
\hline RBC transfusion & 0.001 & 0 & 11.828 & 1 & 0.001 & 1.001 & $1.000-1.001$ \\
\hline Constant & -5.13 & 1.029 & 24.841 & 1 & 0 & 0.006 & \\
\hline
\end{tabular}

Table 7 Intraoperative characteristics (D-dimer value of $1,039 \mu \mathrm{g} / \mathrm{L}$ )

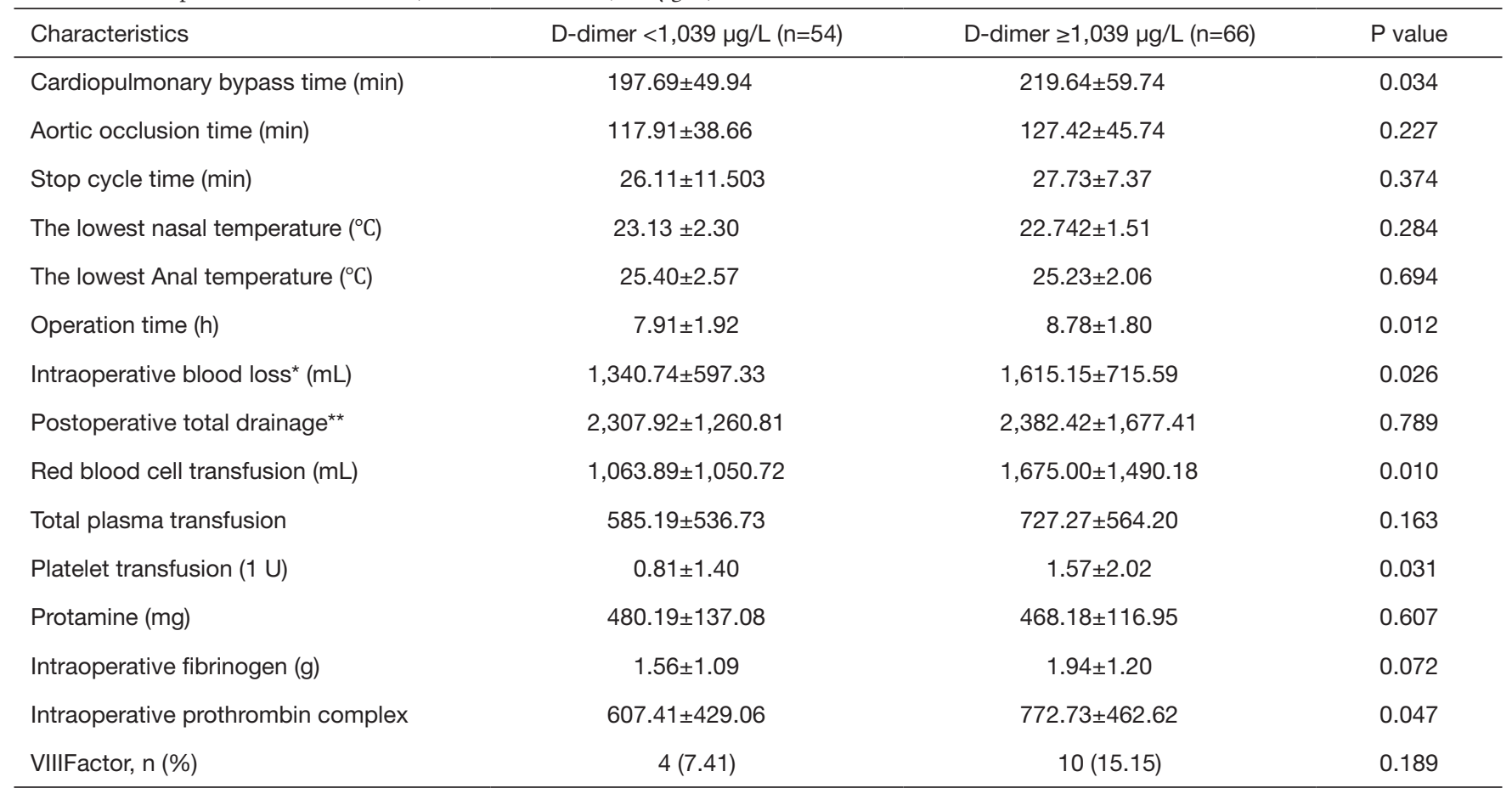

${ }^{*}$, according to the weight of gauze before and after bloodstaining; **, according to intraoperative and after drainage. 
and postoperative bleeding is a major cause of complications. Intraoperative and postoperative bleeding can lead to hemodynamic instability, blood transfusion complications, cardiac tamponade, thoracic bleeding, and even cerebral infarction. There is no reliable way to predict postoperative bleeding; platelet levels, clotting factors, and other parameters change little after anesthetic induction. In this study we investigated that in D-dimer over 3,000 $\mu \mathrm{g} / \mathrm{L}$ groups, the coagulation mechanism was much worse, thus requiring more transfusion and hemostatic treatment (7). Our study population was divided into two groups: those with D-dimer levels less than 3,000 $\mu \mathrm{g} / \mathrm{L}$ and those with D-dimer levels of 3,000 $\mu \mathrm{g} / \mathrm{L}$ or higher (8).

Some patients with type A aortic dissection, in whom blood dissects between the tunica intima and tunica adventitia, will form a large number of thrombi. Many platelets and coagulation factors are consumed in this process, and the fibrinolytic system is activated, which leads to secondary fibrinolysis and eventually to increased D-dimer levels. Does D-dimer elevation suggest that the patient is in a secondary fibrinolytic and coagulation state? In this study, postoperative bleeding, blood transfusion, and use of anticoagulant drugs were higher in Group B than in Group A. The incidence of postoperative acute renal failure was also higher in Group B than in Group A because of greater blood loss intra- and postoperatively.

We selected 3,000 $\mu \mathrm{g} / \mathrm{L}$ as the cut-off point for D-dimer level in this study based on clinical experience and previous studies that used this level as a predictor of risk. Our data confirm that D-dimer levels above 3,000 $\mu \mathrm{g} / \mathrm{L}$ have effects on the coagulation system and renal function. However, there was no significant difference in thromboelastogram between the two groups. These results indicate that D-dimer levels had no effect on thrombosis. This finding may be related to the formation of thrombi in the patient's dissection, the consumption of a large number of coagulation factors, and fibrin degradation. The mechanism will be verified in future animal experiments. Previous experience has shown that a negative D-dimer test is useful in ruling out pulmonary embolism, aortic dissection, and other disorders.

In this study, prognostic value of $\mathrm{D}$-dimer was evaluated using ROC analysis and the results showed that AUC of $\mathrm{D}$-dimer as prognostic indicator for postoperative renal failure was 0.741 (95\% CI, 0.642-0.840; $\mathrm{P}<0.001)$. When a D-dimer value of $1,039 \mu \mathrm{g} / \mathrm{L}$ was used as the cutoff, the corresponding sensitivity and specificity for distinguishing the high risk of dialysis after surgery in patients with Stanford A aortic dissection from low risk were $91.7 \%$ and $54.2 \%$, respectively. Few studies have reported the clinical diagnosis and treatment value of $\mathrm{D}$-dimer concentration. This study revealed the differences of clinical parameters in Stanford A aortic dissection patients after surgery grouped by D-dimer concentration of 3,000 and 1,039 $\mu \mathrm{g} / \mathrm{L}$ (9). D-dimer concentrations above $3,000 \mu \mathrm{g} / \mathrm{L}$ at the time of anesthetic induction predicted greater blood loss in patients with aortic dissection, suggesting that thrombosis and fibrinolytic activity may occur after coagulation system activation. Advance planning in these patients, such as preparation of platelets, plasma, and hemostatic drugs, and streamlining the surgical method to minimize operation time and bleeding, such as retaining arch aortic arch surgery, can reduce postoperative complications (10). In our research, it found that when D-dimer is below $1,039 \mu \mathrm{g} / \mathrm{L}$, the risk of dialysis after surgery in patients with Stanford A aortic dissection is low.

\section{Conclusions}

D-dimer above 3,000 $\mu \mathrm{g} / \mathrm{L}$ increases the risk of dialysis after surgery in patients with Stanford A aortic dissection through the impact of the coagulation system.

When D-dimer is below $1,039 \mu \mathrm{g} / \mathrm{L}$, the risk of dialysis after surgery in patients with Stanford A aortic dissection is low.

\section{Acknowledgements}

We would like to thank Ming Gong and Xiao-Long Wang for preparing the human samples and Jing Liu for their generous support and encouragement during this study.

Funding: This study was supported by National Key R\&D Program of China, High risk warning and intervention for acute aortic syndrome (2017YFC1308000); National Natural Science Foundation of China (81800404 and 81470580); Capital Health Development Research Project (2018-4-2068); Beijing Municipal Administration of Hospitals' Youth Program (QML20180601); Foundation of Beijing Outstanding Young Talent Training Program (2017000021469G254); Beijing Lab for Cardiovascular Precision Medicine (PXM2017_014226_000037).

\section{Footnote}

Conflicts of Interest: The authors have no conflicts of interest 
to declare.

Ethical Statement: This study adopted the consent of the ethics committee of Beijing Anzhen Hospital affiliated to Capital Medical University.

\section{References}

1. Howard DP, Banerjee A, Fairhead JF, et al. Populationbased study of incidence and outcome of acute aortic dissection and premorbid risk factor control: 10-year results from the Oxford Vascular Study. Circulation 2013;127:2031-7.

2. Hughes GC, Zhao Y, Rankin JS, et al. Effects of institutional volumes on operative outcomes for aortic root replacement in North America. J Thorac Cardiovasc Surg 2013;145:166-70

3. Pacini D, Di Marco L, Fortuna D, et al. Acute aortic dissection: Epidemiology and outcomes. Int J Cardiol 2013;167:2806-12.

4. Hugli OW. Letter by Hugli regarding article, "Diagnosis of acute aortic dissection by D-dimer: the International Registry of Acute Aortic Dissection Substudy on Biomarkers (IRAD-Bio) experience". Circulation

Cite this article as: Han L, Dai L, Li HY, Lan F, Jiang WJ, Zhang HJ. Elevated D-dimer increases the risk of dialysis after surgery in patients with Stanford A aortic dissection through the impact of the coagulation system. J Thorac Dis 2018;10(12):6783-6793. doi: 10.21037/jtd.2018.11.138 2010;121:e23; author reply e24.

5. Bossone E, Corteville DC, Harris KM, et al. Stroke and outcome in patients with acute type A aortic dissection. Circulation 2013;128:S175-9.

6. IST-3 collaborative group, Sandercock P, Wardlaw JM, et al. The benefits and harms of intravenous thrombolysis with recombinant tissue plasminogen activator within $6 \mathrm{~h}$ of acute ischaemic stroke (the third international stroke trial [IST-3]): a randomised controlled trial. Lancet 2012;379:2352-63.

7. Yuan SM, Shi YH, Wang JJ, et al. Elevated plasma D-dimer and hypersensitive C-reactive protein levels may indicate aortic disorders. Rev Bras Cir Cardiovasc 2011;26:573-81.

8. Fan QK, Wang WW, Zhang ZL, et al. Evaluation of D-dimer in the diagnosis of suspected aortic dissection. Clin Chem Lab Med 2010;48:1733-7.

9. Shimony A, Filion KB, Mottillo S, et al. Meta-analysis of usefulness of d-dimer to diagnose acute aortic dissection. Am J Cardiol 2011;107:1227-34.

10. Yoshimuta T, Yokoyama H, Okajima T, et al. Impact of Elevated D-Dimer on Diagnosis of Acute Aortic Dissection With Isolated Neurological Symptoms in Ischemic Stroke. Circ J 2015;79:1841-5. 